\title{
Arzanà
}

16-17 (2013)

Écritures de l'exil dans l'Italie médiévale

Cécile Le Lay

\section{« Di questo tempestoso mare stella »}

La Stella maris dans la poésie italienne, religieuse et profane, des XIIle et XIVe siècles

\begin{abstract}
Avertissement
Le contenu de ce site relève de la législation française sur la propriété intellectuelle et est la propriété exclusive de l'éditeur.

Les œuvres figurant sur ce site peuvent être consultées et reproduites sur un support papier ou numérique sous réserve qu'elles soient strictement réservées à un usage soit personnel, soit scientifique ou pédagogique excluant toute exploitation commerciale. La reproduction devra obligatoirement mentionner l'éditeur, le nom de la revue, l'auteur et la référence du document.

Toute autre reproduction est interdite sauf accord préalable de l'éditeur, en dehors des cas prévus par la législation en vigueur en France.
\end{abstract}

revues.org

Revues.org est un portail de revues en sciences humaines et sociales développé par le Cléo, Centre pour l'édition électronique ouverte (CNRS, EHESS, UP, UAPV).

Référence électronique

Cécile Le Lay, « «Di questo tempestoso mare stella » », Arzanà [En ligne], 16-17 | 2013, mis en ligne le 16 juin

2015, consulté le 29 juillet 2015. URL : http://arzana.revues.org/209; DOI : 10.4000/arzana.209

Éditeur : Presses de la Sorbonne Nouvelle

http://arzana.revues.org

http://www.revues.org

Document accessible en ligne sur : http://arzana.revues.org/209

Ce document est le fac-similé de l'édition papier.

Presses Sorbonne Nouvelle 


\section{"Di questo tempestoso mare stella ": la Stella maris dans la poésie italienne, religieuse et profane, des $\mathrm{XIII}^{\mathrm{e}}$ et $\mathrm{XIV}^{\mathrm{e}}$ siècles}

\section{Introduction}

Ses yeux glissèrent sur les sept étoiles du Grand Chariot, les sept étoiles du Petit Chariot: des diamants blanc-bleu dans le noir du ciel. C'était Stella Maris, l'étoile du Nord, l'étoile de la Mer, qui dispensait la lumière la plus aveuglante. Son père, il y avait bien longtemps, avait guidé ses yeux vers cette étoile. Il lui avait appris, en chuchotant une comptine, à lui adresser ses vœux ${ }^{1}$.

Nick Tosches, écrivain et poète américain d'origine irlandaise, connu pour ses biographies de stars du rock (encore des étoiles!), imagine ainsi qu'au milieu des pensées fébriles d'une Athéna moderne qui attend son Ulysse, affleure un lointain souvenir d'enfance, où elle revoit son père lui apprenant à reconnaître l'étoile polaire et à prier celle qu'il appelait encore Stella Maris. La contemplation du ciel semble apaiser pour un temps les craintes de la jeune fille, dont le nom devrait pourtant évoquer la déesse protectrice du héros d'Homère (emblématique pour notre thème lié à la navigatio).

Aujourd'hui, il n'est peut-être plus si simple d'observer les étoiles à l'œil nu, dans nos grandes villes dont les nuits restent baignées de lumières artificielles, et le nom de Stella maris n'évoque peut-être qu'une étoile parmi d'autres (ou même une "étoile de mer", dans une traduction approximative) alors que, pendant des millénaires, l'homme a vécu au contact du firmament : fasciné comme Platon par cette voûte étoilée, il a envisagé son existence comme un

1. Pendant l'été 2008, sur le site du journal Le Figaro, chaque jour on pouvait lire, de la plume d'un grand écrivain étranger, une nouvelle inédite commençant par la même phrase de l'Odyssée : "Ulysse prit le sentier rocailleux qui monte à travers bois, du port vers la falaise. Il allait à l'endroit qu'avait dit Athéna... ». Traduite par Héloïse Esquié, celle de Nick Tosches a été publiée le 30 juillet 2008 : http://www.lefigaro. fr/livres/2008/07/17/03005-20080717ARTFIG00549-nick-tosches-au-dela-desetoiles-.php. 


\section{CÉCILE LE LAY}

temps d'épreuves (d'exil) conditionnant son retour à l'étoile dont il croyait être issu ${ }^{2}$; ou bien, comme Sénèque, il a acquis une telle familiarité avec le ciel qu'aucun lieu terrestre n'était plus capable de le faire se sentir un exilé, le seul redoutable exil consistant à se détacher d'une figure humaine exemplaire et à perdre de vue son idéal stoïcien ${ }^{3}$. Toujours curieux d'en pénétrer les mystères, l'homme a appris très tôt à discerner les signes de la voûte céleste pour orienter son chemin : de fait, en latin, signum a déjà le sens de constellation, et Pétrarque parle des yeux de Laure comme de ses deux dolci usati segni $i^{4}$, que l'on identifie justement avec la Grande Ourse et la Petite Ourse (ou Grand Chariot et Petit Chariot).

Si la dénomination Stella maris pouvait encore être couramment associée à la Vierge Marie au siècle passé, à plus forte raison l'était-elle au XIII ${ }^{\mathrm{e}}$ siècle, au moment où s'affirme la poésie italienne, religieuse et profane, en langue vulgaire, puisque ce fut aussi une période de forte expansion de la dévotion mariale, avec la multiplication des confréries, notamment celles des laudesi, consacrées à l'apprentissage et à la diffusion du chant de laudes en l'honneur de la Sainte Vierge ${ }^{5}$. Cette connotation trop explicite constitue sans doute la raison pour laquelle sa traduction italienne, stella marina, ne figure pas dans la poésie lyrique profane. Elle n'apparaît en effet que dans un contexte religieux, une seule fois d'ailleurs, justement dans l'une des laudes du recueil de Cortone :

Altissima luce col grande splendore, in te, dolze amore, ag[g]iàm consolanza

Ave, regina, pulzell'amorosa, stella marina ke non stai nascosa,

2. Platon, Timée, 41e.

3. Sénèque, Ad Helviam matrem de consolatione, c. IX.

4. F. Petrarca, Canzoniere (Rerum vulgarium fragmenta), M. Santagata (éd.), Milano, Mondadori, 1996 [référence abrégée : RVF], p. 820 : 189, v. 12.

5. La promotion du chant de ces laudes en langue vulgaire eut vraisemblablement son origine à Sienne, à l'initiative du bienheureux Ambrogio Sansedoni († 1268), dominicain entré dans l'ordre dès 1237 . Le décret d'approbation du premier statut de la confrérie de ces laudesi, signé par l'Évêque de Sienne, date de 1267 (les membres d'autres confréries, comme celles des disciplinati, ou flagellanti, nées à Pérouse en 1260, et déjà présentes à Sienne, pouvaient aussi en faire partie). Voir G. G. Meersseman, 1977, Ordo fraternitatis. Confraternite e pietà dei laici nel medioevo, Roma, vol. II, p. 954-964. 
luce divina, virtù grazïosa,

bellezza formosa, di Dio se' semblanza ${ }^{6}$.

À cette citation, on pourrait tout juste ajouter un passage de Pétrarque, tiré de la chanson finale du Canzoniere, explicitement dédiée à la Vierge Marie :

Vergine chiara et stabile in eterno, di questo tempestoso mare stella, d'ogni fedel nocchier fidata guida ${ }^{7}$.

L'hyperbate permet au poète de rapprocher adroitement les deux termes, mare et stella, calquant ainsi le très ancien hymne Ave maris stella, qui date peut-être du $\mathrm{VI}^{\mathrm{e}}$ siècle (son auteur pourrait être Venance Fortunat, $\dagger 609$ ) et a le plus contribué à diffuser cette dénomination mariale ${ }^{8}$.

Bien sûr, depuis les troubadours, en passant par les Siciliens pour arriver en Toscane, la femme a été comparée à une étoile, surtout celle de l'aurore : stella iornaus en provençal, "stella rilucente/ che levi la maitina " pour Giacomo da Lentini ${ }^{9}$; " stella che levi la dia " pour Rinaldo d'Aquino, "sullimata stella de l'albore » chez Pier della Vigna, et « stella de albur » ou "d'albore » de Giacomino Pugliese ${ }^{10}$. Le Mare amoroso renchérit en affirmant : «ben parete dea d'amare, / e meglio che la chiarita stella de la dia ${ }^{11}$ ", mais cette étoile qui apparait si brillante le matin n'est autre que la planète Vénus ${ }^{12}$. Étoile du soir (Vesper) ou étoile du matin (Lucifer), c'est la déesse de l'amour qui est évoquée ou invoquée le plus souvent : point besoin d'aller chercher une quelconque

6. Laudario di Cortona, A. M. Guarnieri (éd.), Spoleto, 1991 [LC], p. 47 : 8, v. 1-6.

7. RVF, p. 1399 : 366, v. 66-68.

8. Parmi de nombreuses occurrences possibles dans la littérature latine médiévale, on peut citer l'Anticlaudianus d'Alain de Lille (V 9).

9. R. Antonelli (éd.), 2008, I poeti della Scuola Siciliana, Milano, Mondadori, "I Meridiani », vol. 1 [PSS 1], p. 339.

10. C. Di Girolamo (éd.), 2008, I poeti della Scuola Siciliana..., vol. 2 [PSS 2], p. 205; $312 ; 633$ et 634 .

11. Il Mare amoroso (v. 134-135), in G. Contini (éd.), 1995 [1960], Poeti del Duecento [PD], vol. 1, t. 1, Milano-Napoli, Ricciardi, p. 492.

12. Dans son étude très détaillée des croisements entre laudes franciscaines et mariales d'une part, et tradition courtoise de l'autre - étude que nous reprendrons plus loin -, Franco Mancini signale que l'ascendance mythique des références bibliques elles-mêmes (cf. Job 38, 7; Isaïe 14, 12 et Apocalypse 22, 16) est désormais bien attestée (F. Mancini, 1996, Il tempo della gioia. Un'interpretazione del Laudario di Cortona, Roma, Archivio Guidi Izzi [Mancini 1996], p. 44, n. 118). 
allusion mariale dans un tel contexte. De plus, les traités de rhétorique comme l'Ars versificatoria de Matthieu de Vendôme († 1286) recommandent de comparer les yeux de la dame aux étoiles ${ }^{13}$, et lorsque, par exemple, Lapo Gianni dit qu'il fut saisi par « li occhi suoi lucenti come stella ${ }^{14}$ ", nul besoin non plus de penser ici à l'intrusion d'une rhétorique sacrée.

Cependant, comme le prouvent les différentes occurrences précédemment citées, le topos dame-étoile présente en réalité un grand nombre de variantes. Le thème de notre colloque va ainsi nous donner l'occasion d'approfondir le sens de certaines d'entre elles, en tenant compte des occurrences similaires transmises par la tradition religieuse, tout en sachant que celles-ci pouvaient à leur tour avoir été inspirées par la poésie profane.

Dans un contexte religieux, en effet, l'appellation stella maris signifie que Marie n'est pas seulement admirée pour sa beauté lumineuse, mais aussi pour sa capacité à guider l'homo viator, exilé au milieu des tempêtes de ce monde, vers le port du salut, le paradis, qui se situe même au-delà de la patrie d'origine, perdue par l'homme à la suite du péché originel, lorsque Adam et Ève sont chassés du paradis terrestre. De fait, la vision chrétienne a grandement contribué à intérioriser le concept de l'exil en comparant la vie à une longue pérégrination vers cette patrie céleste : certains passages de la Bible sont devenus emblématiques, comme la traversée du désert derrière la colonne de feu (dans le cas du peuple hébreu)... ou conduits par une étoile (dans le cas des Mages). Aussi l'image de la stella maris se trouve-t-elle insérée dans le vaste domaine métaphorique de la navigatio, dont les réseaux lexicaux et sémantiques se sont largement répandus dans la littérature religieuse mais ont également foisonné dans la poésie lyrique des origines.

Certains topoi liés à la mer pouvaient bien sûr avoir été directement transmis par la poésie lyrique latine, française et provençale, comme l'image de la tempête, depuis toujours associée à la passion amoureuse, ou bien le jeu de mots entre les trois termes français : «la mer» (l'eau), "l'amer» (le fait d'aimer), et «l'amer » (l'amertume), que l'on retrouvera aussi dans l'avertissement final du Mare amoroso ${ }^{15}$. Toutefois, il est intéressant d'observer que, sur le

13. "Stellis praeradiant oculi " ("les yeux sont plus brillants que les étoiles ») : Matthieu de Vendôme, Ars versificatoria (I, 56, 15), in E. Faral (éd.), 1924, Les Arts poétiques du XII et du XIII siècle, Paris, Champion, p. 129.

14. PD II/2, p. 591.

15. " "Chi vuole amare, li convien tremare, / bramare, chiamare, sì come 'l marinaio in mare amaro;/ e chi no.m crede, mi deg[g]ia mirare per maraviglia,/ ché per amor son morto in amarore, / sì com'è morto Nadriano e Caedino ; però si guardi chi s'ha a guardare" ": Il Mare amoroso (v. 329-334), PD I/ 1, p. 500. 
plan des techniques de navigation, la diffusion et le perfectionnement récents de l'usage de la boussole ${ }^{16}$ ont ajouté de nouveaux éléments métaphoriques à l'image traditionnelle de l'attraction-attirance de l'aimant sur le fer qui illustrait depuis longtemps déjà les rapports entre la dame et l'amant ou la passion amoureuse ${ }^{17}$. La référence à l'étoile polaire se trouve ainsi renforcée.

Notons au passage que l'on appelait communément Maria l'étoile polaire, et non Stella maris. C'est le nom qui apparaît dans certains traités scientifiques comme le De natura rerum de Thomas de Cantimpré ${ }^{18}$, et Dante s'inspire

16. L'invention de la boussole magnétique reste obscure. L’Amalfitain Flavio Gioia, son prétendu inventeur au début du XIV ${ }^{\mathrm{e}}$ siècle, n'a en fait jamais existé, mais sans doute l'avait-on utilisée et perfectionnée pour les besoins de la République Amalfitaine. Vers 1100 son usage par les Chinois est attesté, mais le traité De utensilibus (vers 1187) d'Alexandre Neckham montre qu'elle était aussi utilisée par des marins européens, bien avant que Marco Polo ne revienne de son voyage en Extrême-Orient à la fin du XIII ${ }^{\mathrm{e}}$ siècle. Quant aux Arabes, ils n’en parlent que vers 1220. Dès 1206, Guiot de Provins donne une description précise de l'aiguille aimantée et de ses propriétés, et les fondements du magnétisme furent exposés pour la première fois de façon détaillée en 1269 par Pierre Le Pèlerin de Maricourt, philosophe français et maître de Roger Bacon, dans son Epistola de magnete (Voir "Boussole", in M. Mourre, 1978, Dictionnaire encyclopédique d'histoire, Paris, Bordas, vol. 1, p. 632). Calamita fut utilisée pour indiquer l'aiguille de la boussole en Italie, et c'est aussi le nom donné aux montagnes ferreuses du sud de l'île d'Elbe. Nous reviendrons sur l'emploi de ce terme en poésie.

17. Selon le Physiologos, texte chrétien écrit en grec avant le $\mathrm{IV}^{\mathrm{e}}$ siècle, traduit en latin et maintes fois repris dans les bestiaires et lapidaires du Moyen Âge, l'attraction de l'aimant sur le fer doit être interprétée comme un signe de l'amour encore plus irrésistible de Dieu pour ses créatures : A. Zucker (éd.), 2004, Physiologos : le bestiaire des bestiaires, Grenoble, Millon, $\$ 38$ L'aimant, p. 219-220.

La mythologie permit de diffuser une lecture "érotique " de ce phénomène : en effet, se souvenant du poème Magnes de Claudien ( $†$ 404?), " œuvre ayant connu un certain succès au XII ${ }^{\mathrm{e}}$ siècle [et] qui illustre les propriétés de l'aimant par l'adultère entre Mars et Vénus ", l'auteur de l'Eneas interprète la présence des aimants sur les murailles de Carthage - puisque la réaction physique de l'attraction du fer par l'aimant peut être lue comme la métaphore de l'attirance sexuelle - comme annonciatrice des futures amours d'Eneas et Dido (Virginie Dang, 2001, "De la lâcheté du guerrier à la maîtrise du prince : Eneas à la conquête du pouvoir ", Le Moyen Âge, CVII, p. 11).

Ce phénomène physique sera repris, dans la poésie lyrique romane, comme métaphore des pouvoirs irrésistibles de la dame ou d'Amour sur le cœur du poète. Présente chez les troubadours et les trouvères, elle se retrouve chez les Siciliens, les Toscans, les Stilnovistes et jusque chez Pétrarque.

18. Thomas de Cantimpré en parle dans son De natura rerum (1228-1244?), pour expliquer le fonctionnement de la boussole, dont il donne une description détaillée 
sans doute de cette assimilation lorsque, dans un passage du Convivio, il désigne ainsi la ville hypothétique "tombant" sous le pôle nord ${ }^{19}$. Si l'étoile polaire n'apparaît pas en tant que Maria dans la poésie lyrique profane, en revanche certains poètes l'appellent tramontan ${ }^{20}$, mot qui avait aussi ce sens dans la prose, en plus de celui de "tramontane " ou "vent du nord ", comme le prouve le récit de Marco Polo ${ }^{21}$. Mais peut-être est-ce encore un héritage de la poésie provençale (et française?), puisque ce terme figure chez Sordello et dans le Mare amoroso, ainsi que chez Monte Andrea, Chiaro Davanzati et Guido Guinizzelli ${ }^{22}$.

Pour clarifier notre exposé, nous commencerons par présenter à grands traits l'élargissement des notions liées à la figure de la Vierge comme Stella maris au long de l'histoire de l'Église; puis, dans une seconde partie, nous passerons à l'analyse de notre corpus lyrique, qui s'étend de l'École sicilienne jusqu’à Pétrarque.

qu'il ne reprend cependant pas de sa source habituelle, le De gemmis de Marbode de Rennes (†1123), ce qui induit à penser qu'elle s'est diffusée depuis, donc entre 1123 et 1187, date du premier traité qui en fait mention (le De utensilibus de Neckham) : voir L. Thorndike, 1932, A History of Magic and experimental Science during the first thirteen centuries of our era, vol. 2, p. 190 et 387-388.

19. Convivio, III, V, 9-10.

20. Franco Mancini rapporte à ce propos un vers de Gillebert de Berneville, trouvère d'Artois : «l'estoile qu'on nomme Tremontaine » (Mancini 1996, p. 46, n. 122).

21. Marco Polo, Il Milione, 58, 3; 59, 1; 162, 2; 163, $1 \ldots$

22. De Lollis, Cesare, 1969 [1896], Vita e poesie di Sordello di Goito, Bologna, Forni, XX, p. 178, chanson Aitant ses plus, v. 14-15: «cum la naus en mer guida/la tramontana e 1 fers e lh caramida "; Il Mare amoroso (v. 293-294), PD I/ 1, p. 498 : "sì com' lo marinaro vène a porto/guidandosi per l'alta tramontana »; Monte Andrea da Fiorenza, 1979, Le Rime, F. F. Minetti (éd.), Firenze, Accademia della Crusca [Minetti 1979], p. 89, chanson Tanto miabonda, v. 55-56: « La vera lucë è la tramontana,/e dritta guida de li marinari »; Chiaro Davanzati, 1965, Rime, A. Menichetti (éd.), Bologna, Commissione per i testi di lingua [Menichetti 1965], p. 285, sonnet Madonna, pèré avengna novitate, v. 9 : « ché voi siete del mio cor tramontana "; Guido Guinizzelli, PD II/2, p. 455 et Id., 2002, Rime, L. Rossi (éd.), Torino, Einaudi [Rossi 2002], p. 15-16, chanson Madonna, il fino amor ched eo vo porto, v. 49-50 : «In quella parte sotto tramontana/ sono li monti de la calamita». 


\section{Histoire d'un nom et d'une expression}

Le sixième mois [après la conception d'Élisabeth à un âge avancé], l'ange Gabriel fut envoyé par Dieu dans une ville de Galilée, du nom de Nazareth, à une vierge fiancée à un homme du nom de Joseph, de la maison de David; et le nom de la vierge était Marie (Luc 1, 26-27).

Ainsi commence le récit de l'Annonciation, l'événement qui allait permettre de racheter les hommes en rouvrant les portes du paradis. Rapidement les Pères de l'Église se sont penchés sur la signification de ce nom en apparence si simple : Marie ${ }^{23}$, ou Myriam... Quelque temps avant que le Concile d'Éphèse ne confirme en 431 l'existence de l'union dans le Christ des deux natures, divine et humaine, et ne ratifie le titre de mère de Dieu (Theotokos) attribué à la Vierge Marie, saint Jérôme († 420) avait rassemblé les différentes interprétations de son nom pour identifier la plus juste. C'est ainsi que, dès le début du $v^{\mathrm{e}}$ siècle, l'expression stella maris apparaît la plus appropriée pour en dévoiler le sens caché :

La plupart pensent m'éclairer en interprétant Marie ou bien comme celle qui éclaire, ou bien comme myrrhe de la mer, mais je ne suis pas du tout de cet avis. Il vaut mieux dire qu'elle signifie étoile de la mer, ou bien mer amère, sachant également qu'en syrien Marie est appelée Souveraine [ou Notre Dame] ${ }^{24}$.

L'explication sera reprise au fil des siècles, enrichie de sa justification théologique : c'est parce qu'elle a engendré la lumière du monde, le Seigneur, que Marie est à la fois " étoile de la mer " et "souveraine ", comme le soulignent Isidore de Séville († 636) dans ses Étymologies ${ }^{25}$, et Bède le Vénérable $(† 735)$ dans son Commentaire sur l'Évangile de saint $\mathrm{Luc}^{26}$. Saint Jean Damascène

23. Comme le dit Henri Barré, « pour des gens férus de science étymologique, et cédant volontiers à un penchant prononcé pour le symbolisme [les Pères de l'Église], il était normal de chercher à déceler le sens caché dans ce mot plein de mystère et de grâce : MARIE » : H. Barré, 1939, "La Royauté de Marie pendant les neuf premiers siècles ", Recherches de science religieuse, XXIX, p. 135.

24. "Mariam plerique aestimant interpretari, illuminant me isti, vel illuminatrix, vel smyrna maris, sed mihi nequaquam videtur. Melius autem est ut dicamus sonare eam stellam maris, sive amarum mare : sciendumque quod Maria, sermone Syro, Domina nuncupetur » : J.-P. Migne (éd.), Patrologia latina [PL], 23, 842.

25. PL, 82, $289 \mathrm{~B}$.

26. PL, 92, $316 \mathrm{D}$. 
$(† 749)$ précise et développe la pensée orientale concernant les fonctions de la Vierge, mais sans parler d'étoile :

Regarde vers nous, bonne Souveraine, mère du bon Maître; conduis et dirige nos affaires à ton gré; réprime les assauts de nos mauvais instincts, apaise la tempête et guide-nous vers le port tranquille de la divine volonté; rends-nous dignes de la félicité future ${ }^{27}$.

Cette prière permet d'identifier certains éléments de la métaphore de la vie conçue comme traversée en mer : la tempête correspond aux " assauts de nos mauvais instincts ", ce sont les tentations qui nous mettent en péril, et le "port tranquille » est identifié avec la "divine volonté » vers laquelle Marie peut nous guider, la « félicité future " pouvant être accordée grâce à son intercession. Les interprétations occidentales se réfèrent plus fréquemment à l'étymologie du nom de Marie. À l'époque carolingienne, avec de plus amples développements, nous trouvons celle de Wallafrid Strabon :

Selon ce que pensent la plupart, Marie est interprétée comme celle qui éclaire et étoile de la mer [...], elle qui nous permet, à nous qui accueillons la lumière de la foi et les grâces divines, d'être conduits vers la vision ineffable du Dieu tout puissant, là où, tout joyeux, libérés de toutes les misères de ce monde, nous pourrons enfin régner avec le Christ dans la joie éternelle ${ }^{28}$.

et celle de Sedulius Scotus, qui explicite la comparaison avec les marins :

Étoile de la mer : car, de même que les marins qui rament vers quelque terre choisissent une étoile qui puisse, par sa position et sa clarté, les conduire sans s'égarer; de même, à ceux qui naviguent en ce monde, sainte Marie est donnée comme étoile de la mer pour que, grâce à elle, ils soient en mesure d'être conduits jusqu'au port de la paix éternelle ${ }^{29}$.

27. J.-P. Migne (éd.), Patrologia graeca, 96, 721 A B.

28. Wallafrid Strabon $(† 849)$ moine de l'abbaye de Fulda, précepteur du petit-fils de Charlemagne (si l'homélie In Initium Evangelii S. Matthaei est bien de lui, voir PL, 114, 859) : «Maria, ut plerique aestimant, interpretatur illuminatrix et stella maris [...], per quam lumen fidei et divinae gratiae accipientes ducamur ad ineffabilem Dei omnipotentis visionem, in qua gaudentes, ab omni tristitia saeculi liberati, in aeterna beatitudine regnare cum Christo mereamur. "

29. Sedulius Scotus († vers 858), Explanatio in quatuor evangelistas, Vatican, Palat. 242, p. 8 (In Mat., I, 16), P. F. Moran (éd.), 1864, Essay on the origin, doctrines and 
Au siècle du renouveau monastique, saint Bernard ( $† 1153$ ) nous en offre les commentaires les plus enflammés dans ses sermons sur la Nativité, dont nous ne citons qu'un court extrait :

Elle est cette brillante et merveilleuse étoile qui se lève, glorieuse et nécessaire au-dessus de cet océan immense, dans la splendeur de ses mérites et de ses exemples.

Ô toi, qui que tu sois, qui dans cette marée du monde, te sens emporté à la dérive parmi orages et tempêtes [...], ne quitte pas les feux de cet astre, si tu ne veux pas sombrer dans la bourrasque.

Quand se déchaînent les rafales des tentations, quand tu vas droit sur les récifs de l'adversité, regarde l'étoile, appelle Marie ${ }^{30}$ !

Enfin, au XIII ${ }^{\mathrm{e}}$ siècle, le Miroir de la Sainte Vierge (Speculum beatae Mariae Virginis), célèbre opuscule du franciscain Conrad de Saxe († 1279), anciennement attribué à saint Bonaventure, reprend saint Bernard et complète les raisons de cette appellation en s'appuyant sur trois d'entre elles, sa pureté, sa clarté et son utilité, utilité qui consiste justement à guider les croyants vers la patrie céleste (il assimile également Marie à l'étoile qui a guidé les mages vers l'enfant Jésus) :

Il faut considérer, en troisième lieu, que Marie est pour nous l'étoile la plus utile, parce qu' elle dirige nos pas vers la patrie céleste, bien plus encore, parce qu'elle nous conduit à travers la mer de ce monde à la grâce de son Fils, comme elle nous conduit au paradis; de sorte qu'elle est pour nous cette étoile très brillante, qui nous conduit comme les trois Mages jusqu’à Jésus-Christ. Oui,

discipline of the Early Irish Church, Dublin, p. 236 : «Stella maris : quia sicut nautae in aliquam terram remigantes aliquod sydus eligunt, cuius signo luceque radiante, sine errore possint adduci; ita Sancta Maria in mari huius mundi navigantibus stella maris data est per quam ad portum perpetuae quietis valeant perduci. "

30. "Ipsa, inquam, est præclara et eximia stella super hoc mare magnum et spatiosum necessario sublevata, micans meritis, illustrans exemplis. $\mathrm{O}$ quisquis te intelligis in huius sæculi profluvio magis inter procellas et tempestates fluctuare [...], ne avertas oculos a fulgore huius sideris, si non vis obrui procellis. Si insurgant venti tentationum, si incurras scopulos tribulationum, respice stellam, voca Mariam. "Super missus est, II, $\$ 17$ (PL, 183, 70C-71A), traduction in Saint Bernard de Clairvaux, 1995, Écrits sur la Vierge Marie, Paris, Mediaspaul, p. 74. 
Marie est l'étoile qui nous est fort nécessaire pour nous diriger au milieu des flots de cette vie ${ }^{31}$.

Certains hymnes ont permis de diffuser cette appellation, comme le plus ancien et le plus célèbre, l'Ave maris stella, ainsi que différentes séquences composées pour les grandes fêtes mariales, notamment celles de l'Abbaye SaintMartial de Limoges ${ }^{32}$, célèbres pour leur qualité poétique. Cette abbaye est d'ailleurs citée à propos des liens probables entre rhétorique profane et sacrée au moment de la naissance de la poésie occitane. Toutefois, la notion d'exil sera surtout transmise (même si l'étoile n'y figure pas) par le Salve Regina, probablement composé par saint Bernard lui-même ${ }^{33}$. Cet hymne, qui était récité tous les soirs, évoque les " enfants d'Ève exilés » qui «supplient, gémissent et pleurent dans cette vallée de larmes » qu'est le monde d'ici-bas, dans l'espoir que Marie, considérée comme leur « espérance » et leur " avocate », intercède pour eux afin qu'ils puissent enfin voir Jésus après la mort, lorsque prendra fin leur exil. D'autres hymnes reprendront ces mêmes thèmes après le XIII ${ }^{\mathrm{e}}$ siècle, comme par exemple le $O$ spes mea cara, ( Ô ma chère espérance ») appartenant

31. Conrad de Saxe, 1839, Miroir de la Sainte Vierge, traduit par M. l'Abbé Thivillier, Lyon, Guyot, p. 29. "Tertio considera, carissime, quod Maria est stella utilissima nos ad patriam caelestem dirigendo, immo ducendo nos per mare huius saeculi ad gratiam Filii sui tamquam ad portum paradisi. Unde ipsa est stella illa clarissime fulgens, tre magos ad Christum rectissime ducens. Maria certe est stella illa, quae in fluctibus praesentis vitae valde est necessaria. » : Conradus de Saxonia, Speculum seu salutatio beatae Mariae Virginis ac sermones mariani, P. De Alcantara Martinez (éd.), 1975, Grottaferrata (Roma), Ad Claras Aquas, p. 188.

32. Analecta Hymnica Medii Aevi, G. M. Dreves, C. Blume, H. M. Bannister (éds), 1889, Leipzig [AHMA], vol. VII : In Natale Domini, p. 35; In Nativitate Domini Nostri, p. 52; et In Assumptione Beatae Mariae Virginis, p. 123.

33. "Salve, Regina misericordiae. / Vita, dulcedo et spes nostra, salve. / Ad te clamamus, exsules filii Evae./Ad te suspiramus, gementes et flentes in hac lacrimarum valle. / Eia ergo, advocata nostra, illos tuos misericordes oculos ad nos converte. / Et Iesum, benedictum fructum ventris tui, nobis post hoc exsilium ostende. / O clemens, o pia, o dulcis Maria! » :Salut, ô Reine de miséricorde./ Notre vie, notre consolation, notre espoir, salut! / Enfants d'Ève exilés, nous crions vers vous. / Vers vous nous soupirons, gémissant et pleurant dans cette vallée de larmes. / Avocate, tournez vers nous vos yeux compatissants. / Et, après cet exil, faites-nous voir Jésus, le fruit béni de vos entrailles./Ô clémente, ô miséricordieuse, ô douce Marie!». Pour les discussions sur l'attribution à saint Bernard, voir la préface de J. M. Canal, 1963, "Salve Regina misericordiae". Historia y leyendas en torno a esta antifona, Roma, Ed. Storia e letteratura, p. 10-13. 
au milieu franciscain ${ }^{34}$, qui associe l'image de l'étoile et la notion d'exil dans une invocation pleine de confiance et d'affection, car la Vierge, grâce encore à sa qualité d'avocate, est présentée de nouveau comme la personnification de l'espérance (voir le spes nostra du Salve Regina) ${ }^{35}$.

Tout au long de l'histoire de l'Église, l'interprétation allégorique de la Bible et de certains auteurs de l'Antiquité, comme Ovide et Virgile, a permis d'enrichir cette image de Marie. D'une part elle fut associée à la figure d'Esther, reine juive de l'Ancien Testament, dont le nom signifie aussi étoile en persan (Esther est l'équivalent du prénom latin Stella, et du prénom français Estelle), considérée "à double titre comme une des préfigures de la Stella maris ", parce qu'elle "annonce la Vierge couronnée et médiatrice 36 ". D'autre part elle fut associée à Astrée ('A $\sigma \tau \alpha \alpha^{\alpha} \alpha$ ), nom grec donné à la constellation de la Vierge, qui évoque le temps de l'âge d'or où cette vierge (fille de Zeus et de Thémis, la justice) régnait sur la terre, avant que la méchanceté des hommes ne la fasse remonter au ciel où elle s'est transformée en constellation. Ovide en parle dans les Métamorphoses (I, 149) ${ }^{37}$, et surtout Virgile dans la IVe Églogue (v. 4-10), lorsqu'il annonce son retour et la venue d'un enfant divin : cette églogue fut interprétée comme une prophétie du rôle qu’allait jouer la Vierge Marie dans

34. O. Magnoni (éd.), 1937, Melodie gregoriane e laudi medioevali, Perugia, coll. « Sagra musicale dell'Umbria ", p. 15.

35. "O spes mea cara, Maria praeclara, / e valle amara imploro te./Exaudi praecantem, solare clamantem, / confirma labantem, et serva me.// Per blanda per dura, ad non peritura/o fortis, o pura duc debilem. / Dum turget procella et suscitat bella/ad portum, o stella, duc exulem [...]. " " Ô ma chère espérance, Marie resplendissante, / de cette vallée de douleur je t'implore. / Exauce-moi quand je te prie, consolemoi quand j'appelle, / soutiens-moi quand je vacille, et veille sur moi.// Au travers des facilités ou des difficultés, vers ce qui ne périt pas, / ô toi qui es forte, ô toi qui es pure, conduis-moi quand je suis faible./Tandis que la tempête fait rage et qu'elle déchaîne les conflits (intérieurs), / ô mon étoile, conduis-moi vers le port, moi qui suis exilé [...]."

36. " De même que son couronnement par Assuérus est l'image du Couronnement de la Vierge, son intervention auprès du roi est l'emblème de l'intercession de Marie auprès de son Divin Fils au jour du Jugement : elle avait obtenu la grâce des Juifs, la Vierge obtient la grâce du genre humain »: L. Réau, 1956, Iconographie de l'art chrétien, Paris, PUF, II/1, p. 336.

37. Un petit poème pseudo-autobiographique, le De vetula, qui date en réalité de la première moitié du XIII ${ }^{e}$ siècle (voir M. Picone, 1999, " Dante, Ovidio e la poesia dell'esilio ", Rassegna Europea di Letteratura Italiana, $\mathrm{n}^{\circ}$ 14, p. 8), évoque la conversion tardive de son auteur présumé, Ovide, en terre d'exil, et dit qu'il avait enseigné les mystères du Seigneur, ainsi que la vie et l'Assomption de la Vierge Marie (sans doute pour "compléter et rectifier" l'histoire d'Astrée, remontée elle aussi au ciel). 


\section{CÉCILE LE LAY}

l'Incarnation. Enfin, Marie est souvent comparée à l'étoile du matin pour sa luminosité et pour le fait qu'elle prépare la venue du soleil, son Fils. L'association avec la figure de Vénus était donc fréquente, même dans un contexte religieux. C'est la chiara stella d'oriente ou la lucente stella dïana des laudes de Cortone $^{38}$, qui apparaît encore avec son ancien nom, lucifer ${ }^{39}$, dans une séquence de l'Abbaye de Saint-Martial composée pour la fête de l'Assomption ${ }^{40}$.

\section{$\mathrm{La}$ « stella maris » dans la poésie lyrique}

Pour explorer la vaste étendue de notre corpus, nous allons donc nous concentrer sur les occurrences où figure une étoile (ou constellation) capable d'orienter une traversée en mer, et sur celles où l'aiguille aimantée de la boussole introduit une variante dans le topos courtois de la toute-puissance d'Amour (ou de la dame) comparable à l'attraction irrésistible de l'aimant sur le fer.

Chiaro Davanzati emploie le terme tramontana (sans référence à la boussole) pour désigner sa dame dont il décrit l'influence positive sur son cœur : grâce à elle, c'est en fait toute sa vie qui ne souffre plus d'instabilité, puisqu'elle écarte de sa mente toute menace de fol'amor. L'image traditionnelle

38. LC, p. $67: 14$, v. 10 et p. $95: 20$, v. 8 . La seconde expression apparaît dans l'incipit du sonnet de Guinizzelli, "Vedut'ho la lucente stella diana, / ch'apare anzi che 'l giorno rend'albore » (PD II/2, p. 469, v. 1-2); et «stella diana » figure dans le sonnet "Io voglio del ver la mia donna laudare" du même auteur, au vers 3 : "più che la stella dïana splende e pare " (ibid., p. 472). "Diana " apparaît ici comme un dérivé de "dia ", le " jour ", qui synthétise les expressions relevées chez les Siciliens et dans Il Mare amoroso. "Stella diana » équivaut ainsi à " lucifer ", qui signifie " (étoile) apportant la lumière " : celle qui se lève la première, lorsqu'il fait encore nuit. Pour d'autres références, religieuses et profanes, nous renvoyons à : Mancini 1996, p. 44-45.

39. L'identification entre Lucifer et Satan ne s'imposera que progressivement, multipliant ainsi l'ambiguïté des références (voir A. Pézard, 1949, "Vénus et Dante », in Id., 1975, Dans le sillage de Dante, Paris, Société d'études italiennes, p. 71). C'est un passage d'Isaïe qui sert de justification à cette interprétation, car la satire qui y est faite du roi de Babylone évoque aussi la chute de Satan ("Quomodo cedidisti de cœlo, Lucifer, qui mane oriebaris? » : "Comment es-tu tombé du ciel, Lucifer, qui te levais le matin?", Isaïe, 14, 12).

40. "Oritur/ut lucifer/inter astra/aetherea,/perpulchra ut luna »: "Elle se lève, /comme lucifer/parmi les astres/du ciel,/aussi belle que la lune» (AHMA, vol. VII, p. 122). 
de l'attraction de l'aimant sur le fer réapparaît ensuite, associée à sa beauté et à l'emprise totale que celle-ci exerce sur lui :

ché voi siete del mio cor tramontana, ché non si muta da voi la mia vita e voi amando la mente mi sana. Ed èmi sì vostra bieltà gradita, che mai non parto, sì mi par sovrana, ma corro a ciò com' ferro a calamita ${ }^{41}$.

Mancini cite ces deux tercets pour montrer l'équilibre instable qui persiste entre le « charisme » de la dame et son attraction corporelle dans la poésie profane, tandis que la Vierge Marie des laudes de Cortone, « de li erranti tramontana ", est perçue comme un "guide infaillible et une force qui attire par grâce et non par une loi physique ${ }^{42}$ ».

Un développement plus articulé de cette métaphore de la navigation sous la conduite d'une "bonne" étoile (diritta spera) nous est offert par l'unique sonnet du dénommé Balduccio d'Arezzo, dont on ne sait pas grand-chose. Il se compare ici à un marin sans avoir jamais vu la mer :

Eo son lo marinar ben a ragione,

ed unque il mar, non vidi al mi' scïente;

ed ag[g]io la diritta speragione

c'hanno li marinar comunemente,

senza la qual giriano a tastone,

a guisa che fa l'orbo miscredente,

che davanti si porta lo bastone

e va e pèr' e non crede a la gente.

Similemente 'l marinar non gira :

se non andasse a guida de la stella,

molto acatteria caro lo vïag $[\mathrm{g}]$ io.

Dunqu'è la stella la diritta spera,

ed io veracemente spero 'n ella,

e son lo marinaio che detto $\mathrm{ag}[\mathrm{g}] \mathrm{io}^{43}$.

41. Menichetti 1965, p. 285, sonnet Madonna, pere' avengna novitate, v. 9-14.

42. Mancini 1996, p. 46, n. 122. Voir LC, p. 95 : 20, v. 9.

43. PD I/1, p. 363. 


\section{CÉCILE LE LAY}

Il faut remarquer dans ce sonnet la présence récurrente de la notion d'espérance (à laquelle il est difficile de ne pas associer une certaine connotation mariale, ne serait-ce que par la comparaison avec l'orbo miscredente), grâce aux figures de style construites autour de speragione, spero et spera. Ce dernier terme, qui signifie sphère céleste (au sens de globe, astre), est attiré dans la série pseudo-étymologique autant par le polyptote jouant sur l'homophonie sperol spera que par le parallélisme marqué entre la diritta speragione du vers 3 et la diritta spera du vers 13 : "è la stella la diritta spera ${ }^{44}$ ".

Un sonnet de Monte Andrea da Firenze présente la même métaphore filée (on ne sait lequel des deux sonnets est le plus ancien, probablement celui de Balduccio), mais sans la notion d'espérance. C'est un sonnet qui, à la manière de Guittone d'Arezzo, comporte dix vers à la place des deux quatrains :

Sì come, i marinari, guida la stella, che per lei ciascun prende suo vïag $[\mathrm{g}] \mathrm{io}$;

e chi, per sua follia, si parte d'ella, radoppia tostamente suo danag[g]io :

la mia dritta lumera qual è, quella che guida in terra me e 'l mi' corag[g]io?

Voi, gentile ed amorosa pulzella, di cui m’à mess', a $[\mathrm{h}]$, Amore in sengnorag[g]io !

Ché troppo è scura la mia vita e fella a gir, se vostra lumera non ag[g]io.

La qual fa disparére ongn'altra luce : ché là ove apar vostro angelico viso, altro sprendor giamai non vi riluce. Pulzella, poi mavete sì conquiso che sol per voi mia vita si conduce, merzé, dal vostro amor non sia diviso ${ }^{45}$ !

Dans l'hypothèse, qui reste à vérifier, de l'antériorité du sonnet de Balduccio, Monte Andrea pourrait avoir emprunté aux tercets de l'Arétin la rime -ella ainsi que les deux mots à la rime stella : ella (dans le même ordre), dont

44. Dans le syntagme diritta speragione du vers 3, l'adjectif diritta acquiert, comme le signale Contini, la valeur adverbiale de " exactement "; diritta pourrait ensuite vouloir dire "fiable ", comme l'étoile polaire (ou les étoiles en général, si stella au vers 10 a un sens collectif comme l'indique la note).

45. PD I/1, p. 466; Minetti 1979, p. 154. 
il prolonge la série par quella : pulzella ${ }^{46}$ : fella. Dans ce sonnet, où la navigatio n'est pas évoquée de façon allusivement métaphorique mais comme terme explicite de comparaison, le poète (en "marin de terre ferme ») considère sa dame comme la dritta lumera, capable de le diriger tout au long de son existence in terra. Sans elle, sa vie serait scura et "fella/a gir" (scomoda, selon Contini, mais l'idée de déviation reste présente ${ }^{47}$ ).

La chanson Tanto mabonda du même auteur (strophe IV) constitue pour Mancini un exemple qui se situerait dans la ligne novatrice des laudes de Cortone tendant à "spiritualiser la "vertu" de l'étoile tramontane ${ }^{48}$ " :

46. On peut remarquer la présence de l'expression amorosa pulzella à la rime, puis de pulzella au début du dernier tercet, terme plutôt rare que l'on trouve déjà dans le Contrasto de Cielo d'Alcamo ("Rosa fresca aulentis[s]ima - ch'apari inver' la state/le donne ti disiano, - pulzell'e maritate », v. 1-2, PD I/1, p. 177) et qui provient de la langue d'oïl pulcele (ce dérivé du latin populaire *pu(e)llicella, diminutif de puella - jeune fille -, croisé avec *pullicella, diminutif de pullus - petit d'un animal -, figure dans la fameuse Cantilène ou Séquence de Sainte Eulalie, écrite vers 881 et considérée comme le premier texte poétique en langue d'oïl); il pouvait signifier " jeune fille " (non mariée), « servante ", ou "vierge ", sachant que le trait de "virginité " ne s'affirmera qu'en moyen français. C'est donc surtout l'adjectif d'ascendance courtoise amorosa (" qui suscite l'amour "), présent dans l'expression pulzell'amorosa de la laude de Cortone déjà citée (LC, p. 47 : 8, v. 3), qui pourrait indiquer une influence de la lyrique profane sur la lyrique sacrée.

Inversement, l'influence de la lyrique sacrée sur la lyrique profane pourrait être décelée grâce à un autre terme rare à la rime avec stella, le mot ancella, fortement connoté puisque sa forme latine appartient à l'une des prières les plus répandues à cette époque (l'Angélus, récité trois fois par jour avec la réplique de Marie : «Ecce ancillam Domini »). Présent dans de nombreuses laudes (à la rime avec stella par exemple in LC, laudes 7 et 9), ce mot n'apparaît que "tardivement" dans la lyrique profane : chez Lapo Gianni (ballade Questa rosa novella, v. 24, in PD II/2, p. 591), Dino Frescobaldi (sonnet In quella parte ove luce la stella, v. 5, in id., 1984, Canzoni e sonetti, F. Brugnolo (éd.), Torino, Einaudi, p. 78) et Dante (chanson Amor, che movi tua vertù da cielo, v. 18, in Dante Alighieri, 2005, Rime, D. De Robertis (éd.), Firenze, Edizioni del Galluzzo, p. 84). Grâce à la substitution de l'amant, respectivement par son alma, sa mente, ou son anima, le changement de "genre grammatical" permet que celle-ci soit fatt'ancella de la dame-stella ou d'Amour, dissociant et "laïcisant" ainsi les référents de stella et ancella.

47. Le sonnet 72 de Guittone d'Arezzo, Con più m’allungo, più m’è prossimana, présente certaines similitudes de sens et de rimes, comme dans les tercets (v. 9-14) : "Così como guidò i Magi la stella, / guida sua fazon, gendome avante, / che visibel mi par e incarnat'ella: // però vivo gioioso e ben istante,/ che certo senza ciò crudele $\mathrm{e}$ fella/morte m'auciderea immantenante » (PD I/1, p. 245).

48. Mancini 1996, p. 46, n. 122. 


\section{CÉCILE LE LAY}

La vera lucë è la tramontana, e dritta guida de li marinari;

che troppo fôran lor vïaggi amari, se d'essa la vertute no lgli aita.

Così, de la generazione umana,

e' lum'è, porto, vïa ë ritengno

(e’ fa ciascun dritto, puro e dengno!);

ed anco più : ché presta e tolle vita ${ }^{49}$.

La seule précision qui manque cependant à la lecture édifiante de Mancini concerne l'identification du $e^{\prime}$ anaphorique des vers 60 et 61 . La chanson de Monte se présente en effet comme ayant été inspirée par une matière débordante et irrépressible, qui se manifeste d'abord comme une inspiration amoureuse traditionnelle, mais concerne en fait la toute-puissance de l'argent, comme le déclare déjà la strophe II et le rappelle l'exclamation du vers 63 qui vient tout de suite après le passage cité : " (Ché, Ricorë, e’ quest'è per lo fermo! $)^{50} »$.

Dans un sonnet du Fiore, nous voyons décrite l'habileté nécessaire à « chi d'Amor vuol gioire ", habileté qui consiste à louvoyer entre les états d'âme contraires de la dame, à la façon d'un marin se servant de l'étoile polaire, du vent et des voiles, "insin che 'l mar si va rabonacciando " :

« Il marinaio che tuttor navicando

va per lo mar, cercando terra istrana, con tutto si guid'e' per tramontana, sì va e' ben le sue vele cambiando e per fug[g]ire da terra e apressando, in quella guisa c[h]'allor gli è più sana : così governa mese e settimana insin che 'l mar si va rabonacciando.

Così dé far chi d'Amor vuol gioire quand'e' truova la sua donna diversa :

49. Minetti 1979, p. 89, v. 55-62.

50. Ce qui confirme l'importance de la lettre que Guittone écrit "à un ami ruiné ", identifié avec ce même Monte Andrea. Voir C. Le Lay, 2010, «La consolation par la citation : la lettre de Guittone d'Arezzo à un ami ruiné (lettre III) ", in Écritures et pratiques de l'amitié dans l'Italie médiévale, A. Fontes Baratto (éd.), Arzanà, 13, p. 109-136. 
un'or la dé cacciar, altra fug[g]ire.

Allor sì.lla vedrà palida e persa,

ché sie certan che le parrà morire

insin che no.lli cade sotto inversa ${ }^{51}$."

Le passage du Roman de la Rose correspondant à ce sonnet (v. 7551-7560) ${ }^{52}$ ne précise pas de quelle étoile il s'agit, mais suivre une estoile (v. 7553) signifie qu'elle est unique, par opposition à toutes les "voiles " qu'il faut " changer " pour chercher à atteindre la terra istrana (démultipliée en mainte terre sauvage dans le Roman, v. 7552), qui semble parodier la "terre promise" mais toujours différée du code courtois. Dans le sonnet du Fiore, la référence à l'étoile exclut d'autant plus toute allusion à la beauté resplendissante de la dame, imaginée au contraire comme pallida e persa, que, dans les tercets, la métaphore de la chasse remplace celle de la navigation. Le résultat des manœuvres adroites de l'amant-chasseur se trouve explicité par le vers final ("insin che no.lli cade sotto inversa $)$, alors que Jean de Meun concluait son exemple en restant dans le domaine allusif des intentions initiales: "Qui veut de bonne amour joÿr ». Mais on peut citer, comme autre exemple d'explicitation du port sousentendu par la navigatio courtoise, l'affirmation lapidaire de Guittone, écrite avant sa "conversion" (sans développer aucun élément de la métaphore, donc sans aucune étoile) : " che mai de cosa null'altra mi membra, // che a vedere como porto o riva/ prender potesse intra le vostre membra ${ }^{53}$ ".

Quant à la calamita, son fonctionnement à distance avait déjà inspiré à Guido delle Colonne une variante (sans référence à l'étoile polaire, ou même à la navigation) pour le topos traditionnel associé à l'attraction de l'aimant sur le fer, avec l'introduction d'un troisième terme qui valorise le rôle de la dame : de même que l'aimant ne peut attirer le fer sans que l'air environnant ne le permette, de même Amour ne peut se passer de la femme s'il veut réussir à attirer le poète ${ }^{54}$. Guido Guinizzelli reprend et complète cette image (associée cette

51. G. Contini (éd.), 1984, Il Fiore e il Detto d'Amore attribuibili a Dante Alighieri, Milano, Mondadori, sonnet LVI, p. 114.

52. G. de Lorris et J. de Meun, 1992, Le Roman de la Rose, A. Strubel (éd.), Paris, Librairie générale française, p. 458-461.

53. Guittone d'Arezzo, 1940, Le Rime, F. Egidi (éd.), Bari, Laterza, p. 156, sonnet 35, Gioiosa gioi, sovr'onni gioia gioiva, v. 4-6.

54. Dernière strophe de la chanson Ancor che ll'aigua per lo foco lasse (v. 77-80; 85-88) : "La calamita, contano i saccenti/che trare non poria/lo ferro per maestria,/se nno che ll'aire in mezzo le 'l consenti [...];/Così, madonna mia,/l'Amor s'è 


\section{CÉCILE LE LAY}

fois-ci à la navigation par le biais d'une référence à la technique d'aimantation de la boussole) pour renchérir sur les qualités de la dame :

In quella parte sotto tramontana

sono li monti de la calamita,

che dàn vertud'all'aire

di trar lo ferro; ma perch'è lontana,

vòle di simil petra aver aita

per farl'adoperare,

che si dirizzi l'ago ver' la stella.

Ma voi pur séte quella

che possedete i monti del valore,

unde si spande amore;

e già per lontananza non è vano,

ché senz'aita adopera lontano ${ }^{55}$.

Le comparant est ici constitué par li/ i monti, présents dans fronte et sirma qui développent les qualités respectives de la tramontana et de la dame. La rime stella: quella, bien que baciata, distingue, voire oppose doublement (par la position dans les deux parties distinctes de la strophe et le $M a$ qui les sépare) ces deux termes, dont les référents ne coïncident plus. En rendant compte du phénomène "scientifique" de l'aimantation, le poète précise en quoi il n'est pas exactement superposable au rôle de la dame. En effet, l'action de celle-ci est encore plus efficace puisque elle est dotée, grâce à l'excellence de ses vertus, d'un "magnétisme" permanent qui agit même à distance, contrairement à celui de la tramontana, qui doit se servir chaque fois d'un aimant pour que le sien agisse sur l'aiguille de la boussole.

Dans un contexte qui apparemment occulte toute référence à notre navigatio métaphorique mais qui représente une étape fondamentale dans l'évolution "scientifique" du topos dame-étoile, la célèbre chanson Al cor gentil rempaira sempre amore compare dès la deuxième strophe l'action de la dame sur le cor gentil du poète à celle d'une stella sur une pierre précieuse déjà "purifiée" par le soleil ${ }^{56}$. L'emploi de concepts philosophiques comme celui du passage

apperceputo/che non m'avria potuto/traer a ssé, se non fusse per voi. » (PSS 2, p. 101-102).

55. PD II/2, p. 455; Rossi 2002, p. 15-16 : chanson Madonna, il fino amor ched eo vo porto, v. 49-60. On remarquera stella: quella aux v. 55-56.

56. "Foco d'amore in gentil cor s'aprende/come vertute in petra preziosa,/ che da la stella valor no i discende/anti che 'l sol la faccia gentil cosa;/ poi che n'ha tratto 
de la puissance à l'acte, ainsi que la scène finale qui se déroule plaisamment devant le tribunal céleste pour rappeler que certains attributs auraient dû être réservés " a la reina del regname degno " (v. 56), nous montrent l'ambivalence de cette étoile dont les vertus s'apparentent à des "grâces" permettant d'obtenir le beato compimento (v. 46).

C'est encore la technique de la navigation et les progrès rendus possibles par l'utilisation de la boussole qui permettent à Monte Andrea d'ajouter une nouvelle variante au binôme aimant-fer. Il observe en effet qu'après avoir été aimantée la calamita (l'« aiguille » de la boussole) s'oriente aussitôt vers l'étoile polaire et qu'elle continue à indiquer le nord, même lorsque l'étoile est cachée par les nuages :

Poi che 'l ferro la calamita sag $[\mathrm{g}] \mathrm{ia}$ ver' la stella dirizza mantenente, e se la stella, per scurtate ch'ag[g]ia, si cela, già non parte di neiente.

Così, donna, mostrandovi salvag[g]ia, celando me vostra fazzon piagente, non v'ascondete sì, ched io non ag[g]ia agli oc[c] hi vostra figura presente;

e non vi val, ché già non mi scorag[g]ia di voi amar, ma più son ubidiente.

E pur venisse che mi foste ascosa, non vi potesse cogli oc[c]hi cernire, sacciate che lo cor non vi dà posa : giamai non parto voi sempre voi seguire, fintanto che sarete sì pietosa, che non vi piaccia ch'io deg[g]ia morire ${ }^{57}$.

La reprise de Guinizzelli n'est que partielle car, pour celui-ci, sa dame exerce un tel pouvoir d'attraction qu'elle ne nécessite aucune aide intermédiaire. Mais la remarque sur les nuages qui n'entravent plus la navigation à la boussole (celle-ci peut désormais se dérouler par temps couvert, de jour comme de nuit) permet à Monte Andrea d'affirmer que son cœur aussi est

fòre / per sua forza lo sol ciò che li è vile, / stella li dà valore : / così lo cor ch'è fatto da natura / asletto, pur, gentile, / donna a guisa di stella lo 'nnamora » : PD II/2, p. 461, v. 11-20; Rossi 2002, p. 34-35.

57. PD I/1, p. 467; Minetti 1979, p. 268. 


\section{CÉCILE LE LAY}

aimanté comme cette aiguille, puisqu'il peut continuer à suivre des yeux la figura (mentale, comme chez Giacomo da Lentini) de la dame alors même qu'elle lui dérobe sa fazzon.

Dans tous les exemples qui précèdent, c'est donc en fonction de sa dameétoile que le poète décide d'orienter son errance et parfois sa vie, dès lors que la navigation amoureuse se fait métaphore existentielle; mais la voix de Fra Guittone s'élève alors comme un avertissement et un défi car à ses yeux la stella des Fidèles d'Amour n'est plus qu'une "idole» trompeuse ${ }^{58}$, comme la Richesse pour son disciple et ami Monte :

Ma chi cantare vole e valer bene, in suo legno a nochier Diritto pone, ed orrato Saver mette al timone, Dio fa sua stella e ver Lausor sua spene; ché grande onor né gran ben non è stato conquistato, - carnal voglia seguendo, ma promente valendo, ed astenendo - a vizi ed a peccato ${ }^{59}$.

Dans ce cas, la navigatio ne concerne plus seulement la vie du poète, mais son écriture elle-même, qui cherche elle aussi à orienter son inspiration pour atteindre un but ultime. La conscience de la distance et des difficultés peut alors faire apparaître la nécessité d'une nouvelle " étoile » capable de guider le poète dans cette autre traversée. Par le biais d'une "conversion" proclamée et affichée, Guittone semble creuser une ligne de démarcation infranchissable entre inspiration profane et sacrée, mais cela lui permet aussi de récupérer/resémantiser les métaphores courtoises, dont la navigatio et l'étoile, dans la nouvelle optique. Leur malléabilité est confirmée par la persistance de ce champ sémantique dans les Rerum vulgarium fragmenta, où Pétrarque contribue à illustrer l'aspiration à une "conversion" (en tant qu'"exil" voulu, volontaire, sans retour, de tout ce qui l'éloigne de la dritta via) jamais aboutie.

58. En effet l'auteur "converti" a décidé de renier Amour, "ché 'n tutte parte ove distringe Amore / regge follore - in loco de savere » (PD I/1, p. 214, chanson Ora parrà s'eo saverò cantare, v. 10-11).

59. Ibid., p. 214-215, v. 16-23. 
Dans la chanson 73, par exemple, apparaissent les deux constellations qui lui sont chères (strophe IV) ${ }^{60}$ :

Come a forza di vènti

stanco nocchier di notte alza la testa

a’ duo lumi ch'à sempre il nostro polo,

così ne la tempesta

ch'i' sostengo d'Amor, gli occhi lucenti

sono il mio segno e 'l mio conforto solo ${ }^{61}$.

Tandis que le poète est aux prises avec une tempête dont Amour est rendu responsable, son regard reste attiré par les yeux de Laure, son guide et son unique réconfort. Remarquons qu'il ne s'agit plus ici de "l'étoile", singulière ou collective, car elle apparaît sous forme de double lumière (duo lumi), et que la comparaison avec le nocher introduit une nouvelle précision : elle concerne l'attitude du marin et pourrait être une réminiscence de Virgile ${ }^{62}$, mais c'est l'axe de l'attirance, vertical et orienté vers le haut dans ce cas, qui va nous intéresser. De fait, en précisant qu'il se compare au nocher qui « lève la tête » pour se tourner vers les étoiles qui ne disparaissent jamais du ciel (polo), Pétrarque rend explicite ce qui restait sous-entendu dans les autres occurrences de stella (y compris dans la célèbre supplication inlassablement répétée par saint Bernard : Respice stellam, voca Mariam, "Tourne-toi vers l'étoile, invoque Marie »), à savoir que le secours vient d'en haut. Cette remarque devient plus évidente encore lorsqu'il s'agit comme ici d'étoiles "polaires", puisque la trajectoire de l'autre stella fréquemment citée, Vénus, reste toujours relativement basse à l'horizon.

Par opposition à cette verticalité impliquant une connotation positive, l'attraction peut s'exercer vers le bas et conduire au naufrage : deux exemples permettent d'illustrer cette autre variante. Tout d'abord chez Monte Andrea :

60. Comme l'indique la note au vers 48 : « alle due costellazioni dell'Orsa, che mai tramontano nel cielo (polo) settentrionale" (RVF, p. 387). Ce sont "i duo mei dolci usati segni » du célèbre sonnet 189 déjà cité, que nous reprendrons plus loin.

61. Ibid., p. $381: 73$, v. 46-51.

62. La note aux vers 46-48 dit en effet : "varia il paragone tradizionale [...] delle stelle che guidano i naviganti, spostando l'attenzione sul nocchiere, forse per suggestione del Palinuro virgiliano, che "oculos ... sub astra tenebat" (Aneis V 853) " (ibid., p. 386-387). 


\section{CÉCILE LE LAY}

Ma 'n tanto, Amor, la mia morte è più grave :

ch'aggio ongnor morte, e mantengno vita!

Ed e'll veder non sò la mia fenita.

Così Amor condotto m’à a reo passo,

ed in mar tempestoso messo m’àve,

che mi trae a ssé con' ferro calamita!

[...] ma sto in disparte da Lucente Spera

e pur sofrango in pene ${ }^{63}$.

Amour est ici aussi tenu pour responsable de la tempête que le poète doit affronter, et celui-ci tente en vain de se tenir à distance de sa Lucente Spera car un curieux phénomène se produit : la mer semble vouloir l'engloutir comme s'il était attiré par un aimant caché dans les profondeurs. Puis ce phénomène est repris et amplifié par Pétrarque (sans faire référence à l'étoile). Certains récits parlaient en effet de la présence de roches magnétiques au fond de l'océan Indien qui exerçaient une telle force d'attraction sur toutes les parties en fer des navires qu'elles les arrachaient de la coque et les faisaient sombrer :

Una petra è sì ardita

là per l'indico mar, che da natura

tragge a sé il ferro e 'l fura

dal legno, in guisa che' navigi affonde.

Questo prov'io fra l'onde

d'amaro pianto, ché quel bello scoglio

à col suo duro argoglio

condutta ove affondar conven mia vita :

così l'alm'à sfornita

(furando 'l cor che fu già cosa dura,

et me tenne un, ch'or son diviso et sparso)

un sasso a trar più scarso

carne che ferro. O cruda mia ventura,

che 'n carne essendo, veggio trarmi a riva

ad una viva dolce calamita ${ }^{64}$ !

Dans cet exemple, Pétrarque redistribue, de façon diversa et nova (cf. incipit), les composantes liées à la calamita. C'est d'une part l'orgueil impitoyable

63. Minetti 1979, p. 38-39, chanson Ai, Deo merzé, strophe III, v. 37-42; 50-51.

64. $R V F$, p. $653: 135$, v. $16-30$. 
de Laure qui est souligné ici par la comparaison avec cette pierre particulière (scoglio et sasso renvoient à petra), et d'autre part ses pouvoirs destructeurs exceptionnels puisque, plus avide de "chair " que de "fer", elle a réussi à s'emparer du cœur du poète en arrachant la cuirasse qui le protégeait. La vienavigatio de l'amant aliéné risque ainsi d'être conduite à sa fin (riva), engloutie dans les eaux profondes par cette viva - dolce calamita (unie à riva par une rime interne) qui semble l'avoir envoûté.

Dans la chanson 73 déjà citée, le poète affirme ne pas avoir d'autre réconfort que les yeux de Laure dans la tempête qu'Amour déchaîne contre lui. Dès la première strophe, il avait pourtant cherché à échapper à son destin, qui semble le contraindre à s'épancher dans ses poésies, en demandant à ce même Amour de le guider quand il écrit (" sia la mia scorta, e 'nsignimi 'l camino ", v. 5), afin d'éviter que son ardeur amoureuse ne soit trop ravivée par la beauté des vers. La fin de cette strophe présentait en effet l'image redoutée du poète "fondant" sous le charme de ses propres paroles (v. 14-15) : " mi struggo al suon de le parole, / pur com'io fusse un huom di ghiaccio al sole ${ }^{65}$ ".

À la fin de la première partie du recueil Chigi, il a placé un autre sonnet significatif pour notre étude. L'Amour est à la barre et se comporte encore en ennemi. Tous les éléments se liguent contre le poète, mais cette fois-ci il a perdu ses deux points de repère habituels, "i duo mei dolci usati segni ", et finit par désespérer d'atteindre le port (qui devrait être le port du salut éternel) :

Passa la nave mia colma d'oblio per aspro mare, a mezza notte il verno, enfra Scilla et Caribdi; et al governo siede 'l signore, anzi 'l nimico mio.

A ciascun remo un penser pronto et rio che la tempesta e 'l fin par ch'abbia a scherno;

la vela rompe un vento humido eterno di sospir', di speranze et di desio.

Pioggia di lagrimar, nebbia di sdegni bagna et rallenta le già stanche sarte, che son d'error con ignorantia attorto. Celansi i duo mei dolci usati segni; morta fra l'onde è la ragion et l'arte, tal ch'incomincio a desperar del porto ${ }^{66}$.

65. Ibid., p. $380: 73$, v. 1-15.

66. Ibid., p. 820 : 189. 


\section{CÉCILE LE LAY}

Dès le premier vers, Pétrarque déclare qu'il est tombé dans une sorte d'inertie, l'oblio, qui finit par avoir des conséquences profondément néfastes : " [le sarte] che son d'error con ignorantia attorto " (v. 11). Mais le poète semble indiquer l'impossibilité de se repérer sur sa double constellation habituelle comme la cause du "naufrage" de sa raison et de sa capacité à gouverner sa vie (arte, v. 12). Cette confusion est peut-être encore plus visible dans au moins deux autres sonnets se rapportant à la navigation ${ }^{67}$ (le deuxième appartient à la partie dite in morte) :

Fra sí contrari vènti in frale barca

mi trovo in alto mar senza governo,

sí lieve di saver, d'error sí carca

ch'i' medesmo non so quel ch'io mi voglio ${ }^{68}$.

Veggio fortuna in porto, et stanco omai

il mio nocchier, e rotte arbore et sarte, e i lumi bei che mirar soglio, spenti ${ }^{69}$.

Le Canzoniere se termine par la longue chanson dédiée à la Vierge Marie, dont nous avons déjà parlé car l'hymne Ave maris stella y est clairement évoqué au début de la strophe VI. Le poète s'en remet à cette figure rassurante (" chiara et stabile in eterno ») car il se sent seul, abandonné de tous. Toutefois ce recours in extremis à l'intercession de la Vierge ne peut apaiser la tempête qui fait rage, reflet du dissidio qui continue à l'habiter :

Vergine chiara et stabile in eterno, di questo tempestoso mare stella, d'ogni fedel nocchier fidata guida, pon'mente in che terribile procella i' mi ritrovo sol, senza governo, ed ò già da vicin l'ultime strida. Ma pur in te l'anima mia si fida, peccatrice, i' nol nego,

67. Pour de nombreux autres exemples, liés aux images croisées (ou fondues dans le cas de la sextine 80, "Chi è fermato ", ibid., p. 410-411) de la navigation d'amour et de la vie humaine, voir C. Perrus, 2000, "La sextine LXXX du Canzoniere ", Chroniques italiennes, 61, p. 5-15.

68. RVF, p. $642: 132$, v. $10-13$.

69. Ibid., p. $1097: 272$, v. 12-14. 


\author{
Vergine, ma ti prego \\ che 'l tuo nemico del mio mal non rida : \\ ricorditi che fece il peccar nostro \\ prender Dio, per scamparne, \\ humana carne al tuo virginal chiostro ${ }^{70}$.
}

Outre le très célèbre Ave Maris Stella repris textuellement dans le syntagme mare stella, le seul mot qui présente la même rime, procella, pourrait se référer à un autre hymne latin de la tradition mariale, le $O$ spes mea cara, qui fait rimer stella avec procella et bella, dans un même contexte d'exil, de tempêtes et de souffrances : ce n'est sans doute pas un hasard si c'est procella qui termine la série des rimes -ella!

\title{
Conclusion
}

Cherchant à identifier les traces éventuelles d'une Stella maris d'ascendance mariale dans la poésie lyrique italienne profane des XIII ${ }^{e}$ et XIV ${ }^{e}$ siècles, nous avons rapidement constaté que les références religieuses pouvaient être éclipsées par d'autres références littéraires plus immédiates (mythologiques et courtoises). Toutefois, en retraçant les grandes lignes de l'histoire de cette appellation mariale et en nous basant sur les conceptions très répandues d'homo viator et de navigatio liées à notre thème de l'exil (agrémentées de quelques techniques de navigation récemment prises en compte par les poètes), nous avons pu établir des parallèles et des distinctions capables d'enrichir la compréhension de certaines occurrences du topos dame-étoile.

La beauté resplendissante de la dame, illustrée par les multiples variantes de l'étoile du matin (de la Provence à la Toscane en passant par la Sicile), peut ainsi prendre occasionnellement le rôle d'étoile polaire, perçue comme fiable par le poète aux prises avec la tempête amoureuse et cherchant le plus souvent à n'atteindre qu'un port très provisoire; mais les pouvoirs d'attraction de la dame sur le cœur du poète (topos de l'aimant attirant le fer) risquent également de conduire au naufrage (Monte Andrea et Pétrarque). Ce risque, définitivement écarté par Guittone après sa "conversion", continuera d'alimenter les Rerum vulgarium fragmenta, même lorsque la séparation des rôles sera clairement établie entre « i duo [suoi] dolci usati segni » et la Stella maris.

70. Ibid., p. $1399: 366$, v. 66-78. 